\title{
Fluid structure interaction based structure stress and modal analysis of a flat type solar panel supporting system
}

\author{
Handai Liu, Piao Zhang, Zhenqiang Lai, Xiaofeng Dong, Zhicheng Wang, Shumin Zhou \\ Jiangxi Province Engineering Research Center of New Energy Technology and Equipment, East China University of Technology, \\ 330013, Nanchang, China
}

\begin{abstract}
Solar panel supporting systems are often affected by strong wind loads, especially in typhoonintensive areas such as Southeast Asia. This paper focuses on the study of flat-panel solar energy system. The numerical model of solar panel supporting system has been built by using the fluid flow control equations. Firstly, the numerical simulation of aerodynamic characteristics of the solar panel supporting system are conducted, and then the pressure results gained from CFD are loaded and coupled to the front and back of the solar panels. Lastly, the stress, strain and the modal analysis results of the support system under four different directional wind loads are achieved. The conclusions include: (1) under the condition of same wind speed, the wind pressure and distribution of the solar panel are different with different wind directions, and the wind load perpendicular to the solar panel has the greatest influence on the solar supporting system. (2)The modal frequency of flat panel solar supporting system is little affected by wind directions and average wind pressure. In the structure design, low order vibration should be considered mainly in the supporting part of the system, while the high order vibration should be generally considered in vibrating risk of solar panel. (3)The first six modal frequencies coupled with fluid-structure interaction of the flat panel solar supporting system are all slightly lower than the free modal frequencies.
\end{abstract}

\section{Introduction}

Compared with traditional energy, solar energy as a renewable energy has a wide range of applications and significant benefits, and has broad prospects for development [1]. Southeast Asia, Africa and other regions have rich available solar energy resources, and in some of these underdeveloped areas there is a serious power shortage problem. Thus it can be seen that distributed solar power generation system is an effective and feasible method to solve the problem of power shortage. Distributed solar power generation systems are generally installed in sunny places like open flat sites and the tops of high-rise, long-span and low-rise houses [2]. Wind load is one of the main natural damage factors to solar power generation supporting systems, especially in Southeast Asia. Moreover, natural gusts also have the potential to severely damage solar supporting systems. Generally, in the design process of flat panel solar supporting structure, it is usually assumed that the solar panel is subjected to uniform wind force in the wind load calculation, and the wind load is generally not considered in the modal analysis process or just implement the modal analysis of the flat panel system under uniform wind load. Obviously, these assumptions are inconsistent with the actual wind load that solar panel systems are subjected to, so there are large errors and certain risks in relevant design and calculations.

In order to calculate and analyze the flat solar panel supporting system more accurately, it is necessary to calculate the wind distribution on the solar panel under wind load, and then conduct structural stress and modal analysis to optimize the structure of the supporting system. Relatively accurate numerical simulation results can be obtained by interaction analysis between two or more physical fields (multi-physical field coupling analysis) [3]. Wind tunnel test is a reliable method to test and calculate wind load. However, it has disadvantages such as high cost and time consuming, which limits the wide use of this method. It is usually used in the research of aerodynamics in important fields like aerospace engineering. With the development of computer science and technology, the numerical simulation method has become a widely used, efficient, low cost, high accuracy research method of fluid, structural stress and strain. The numerical results of aerodynamic characteristics and structural stress of the physical model under wind load can be obtained quickly and accurately through this method. It can reduce the experimental errors caused by neglecting details or other factors in wind tunnel test. Scholars at home and abroad have also proposed a variety of wind load simulation methods [4-5].

In this paper, the physical model of the flat-panel solar supporting system is reasonably simplified, and the fluid-structure coupling stress and modal analysis under 
different wind load conditions are carried out to obtain the wind pressure distribution, stress and strain of the solar panel and the 6 order modal analysis results. It provides a favorable theoretical basis for its structure optimization and operation maintenance.

\section{The physical model of flat type solar panel supporting system}

The research object of the flat type solar panel supporting system mainly includes fixed seat, supporting columns, inclined beams, transverse beam columns, solar panels and various bolts, various blocks and so on. In order to simplify the physical model, reduce the number of mesh and improve the feasibility of numerical calculation, structural features such as fixed seat, bolts and press blocks are ignored in the establishment of structural model. The simplified physical model consists of four columns and eight solar panels mounted on four beams at a $15^{\circ}$ Angle, as shown in Figure 1.

The length, width and height of the flat type panel solar power generation supporting system is $3.2 \mathrm{~m} \times 4.235 \mathrm{~m} \times 2.98 \mathrm{~m}$. Compared with the solar panels, the support part receives less wind loads, thus the supporting part is ignored in the numerical analysis of the flow field. In order to ensure that the flow of airflow in the computational fluid domain is fully developed and take into account the calculation economy, the size of the computational fluid domain is determined to be $20 \mathrm{~m} \times 10 \mathrm{~m} \times 7 \mathrm{~m}$ after repeated modeling and simulation verification, and the distance between the wind speed inlet and the model center is $7 \mathrm{~m}$, as shown in Fig. 1 .

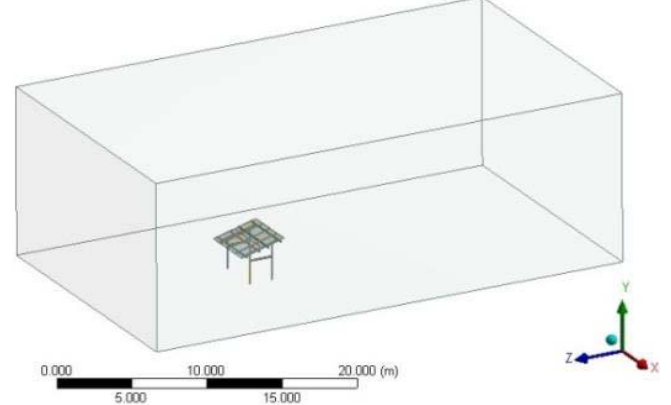

Fig.1. Physical model of flat type solar supporting system

In the flow field calculation domain, air is regarded as a continuous, viscous and uncompressible fluid. The type and number of mesh have a great influence on the accuracy of the calculation results. The polyhedral mesh is used for the fluid region of this model, and the unstructured mesh has good flexibility and adaptability, which is easy to carry out mesh adaptation. In order to simulate the characteristics of the flow field around the solar panel more accurately, the area around the solar panel is treated with grid encryption.

\section{The numerical model of flat type solar panel supporting system}

\subsection{Governing equations of aerodynamics}

Fluid flow should follow the laws of physical conservation, and the basic conservation laws include the laws of mass conservation, momentum conservation and energy conservation. If the fluid contains a mixture of other components, the system should also obey the law of components conservation. The governing equations of the general incompressible Newtonian fluid are described as follows.

Mass conservation equation:

$$
\frac{\partial \rho_{f}}{\partial \tau}+\nabla \cdot\left(\rho_{f} v\right)=0
$$

Where, $\tau$ is time, $s ; \nabla$ is the Hamiltonian operator. $\nabla=(\partial / \partial \mathrm{x}) \mathrm{i}+(\partial / \partial \mathrm{y}) \mathrm{j}+(\partial / \partial \mathrm{z}) \mathrm{k} ; \rho_{\mathrm{f}}$ is fluid density $\mathrm{kg} / \mathrm{m}^{3}, \boldsymbol{v}$ is fluid velocity, $\mathrm{m} / \mathrm{s}$.

Momentum conservation equation:

$$
\frac{\partial \rho_{f} v}{\partial \tau}+\nabla \cdot\left(\rho_{f} v v-\tau_{f}\right)=f_{f}
$$

Where, $\mathrm{f}_{\mathrm{f}}$ is the volume force, $\mathrm{kg} /\left(\mathrm{m}^{2} \cdot \mathrm{s}^{2}\right)$; $\tau_{\mathrm{f}}$ is the shear stress, $\mathrm{P}_{\mathrm{a}}$, which can be expressed as:

$$
\boldsymbol{\tau}_{f}=-\left(\mathrm{p}+\frac{2}{3} \mu \nabla \cdot v\right) I+2 \mu \boldsymbol{e}
$$

Where, $\mathrm{P}$ is the fluid pressure, $\mathrm{P}_{\mathrm{a}} ; \mu$ is dynamic viscosity, $\mathrm{P}_{\mathrm{a}} \cdot \mathrm{s}$; $\mathrm{I}$ is the unit tensor; $\mathrm{e}$ is the velocity stress tensor, $\mathrm{e}=\left(\nabla \mathrm{v}+\nabla \mathrm{v}^{\wedge} \mathrm{T}\right) / 2$ 。

There is no energy generation and transfer in the fluid-structure coupling fluid simulation in this study, so the energy governing equation is not considered.

\subsection{Fluid-structure coupling equation}

In the fluid-structure coupling region, the pressure applied by wind loads on solar panels can cause them to deform. The solar panels have a great influence on the flow velocity and pressure distribution of the upstream and downstream airflow around it. The calculated flow field pressure should be loaded onto the front and rear surfaces of the solar panels, and the fluid governing equation and the solid governing equation should be solved coupling.

The fluid-structure coupling of the flat type solar panel supporting system follows the basic conservation laws. At the fluid-solid coupling interface, the fluid stress $\tau_{f}$ is equal to the solid stress $\tau_{s}$, the fluid displacement $d_{f}$ is equal to the solid displacement $d_{s}$, the fluid heat flow $q_{f}$ is equal to the solid heat flow $q_{s}$ and the fluid temperature $T_{f}$ is equal to the solid temperature $T_{s}$, namely the following four equations [6]:

$$
\left\{\begin{aligned}
\tau_{f} \cdot \boldsymbol{n}_{f} & =\tau_{s} \cdot \boldsymbol{n}_{s} \\
d_{f} & =d_{s} \\
q_{f} & =q_{s} \\
T_{f} & =T_{s}
\end{aligned}\right.
$$

Where, $n_{f}$ is the normal unit vector of the fluid at the fluid-solid coupling interface; $\boldsymbol{n}_{s}$ is the normal unit vector of solid at the interface of fluid-solid coupling. 


\subsection{Fluid-solid coupled modal analysis theory}

The modal frequency of the structural system can be obtained by modal analysis, which provides a theoretical basis for the optimal design of the structural dynamic characteristics. The modal theoretical computing process is as follows:

(1) Free modal: modal analysis of the solar panel supporting system without external loads. The flat-plate solar supporting system is a multi-degree-of-freedom system. For this system, the dynamic solution equation of its elastic structure can be deduced from D 'Alembert's principle [7]:

$$
[\mathrm{M}]\{\ddot{\mathrm{u}}\}+[\mathrm{C}]\{\dot{\mathrm{u}}\}+[\mathrm{K}]\{\mathrm{u}\}=[\mathrm{F}]
$$

When the load is zero, the free vibration equation is:

$$
[\mathrm{M}]\{\mathrm{u}\}+[\mathrm{C}]\{\dot{\mathrm{u}}\}+[\mathrm{K}]\{\mathrm{u}\}=0
$$

(2) Fluid-structure coupled modal analysis: here is the structure modal analysis based on fluid-structure coupled wind load. The vibration equation of fluid structure interaction analysis [8] is:

$$
\left[\begin{array}{cc}
\mathrm{M} & 0 \\
\rho_{\mathrm{f}} \mathrm{R} & \mathrm{M}_{\mathrm{f}}
\end{array}\right]\left\{\begin{array}{l}
\mathrm{u} \\
\mathrm{p}
\end{array}\right\}+\left[\begin{array}{cc}
\mathrm{C} & 0 \\
0 & \mathrm{C}_{\mathrm{f}}
\end{array}\right]\left\{\begin{array}{l}
\dot{\mathrm{u}} \\
\dot{\mathrm{p}}
\end{array}\right\}+\left[\begin{array}{cc}
\mathrm{K} & 0 \\
0 & \mathrm{~K}_{\mathrm{f}}
\end{array}\right]\left\{\begin{array}{l}
\mathrm{u} \\
\mathrm{p}
\end{array}\right\}=\left\{\begin{array}{l}
\mathrm{F} \\
0
\end{array}\right\}
$$

Where, $\mathbf{M}, \mathbf{K}$ and $\mathbf{C}$ are the mass matrix, stiffness matrix and damping matrix of the structure respectively. The subscript $f$ is fluid; $u$ is the displacement vector of structural vibration; $\rho_{\mathrm{f}}$ is fluid density. $\mathbf{R}$ is the fluidstructure coupling matrix; $\mathbf{P}$ is the fluid pressure vector; $\mathbf{F}$ is the dynamic load vector of structural nodes.

\section{4 simulation setting}

\subsection{Numerical simulation setting of flow field}

The main load source of solar panel supporting system is natural strong winds, and the supporting structure is subjected to small wind load, so it is ignored in the fluid simulation. The solar panel supporting system model is imported into ANSYS Workbench, and the support part is restrained after that. Then, the appropriate fluid domain is created, the Boolean operation is carried out, and the mesh is divided. In the fluid simulation of the solar panel supporting system, the energy change is not considered as the fluid temperature change is negligible. Setting of boundary conditions: The wind flow through the solar panel supporting system belongs to turbulence, so the Standard K-epslion model in turbulence model is selected. The fluid is incompressible air with a constant density of $1.29 \mathrm{Kg} / \mathrm{m}^{3}$. Simple algorithm and steady-state solver are applied to solve the case.

1) Inlet conditions: as the conditions of severe weather (force 13 wind), the inlet wind speed is $v=41 \mathrm{~m} / \mathrm{s}$, the temperature is normal and adiabatic, and the reference pressure is 1 standard atmosphere.

2) Outlet condition: the pressure outlet boundary condition is adopted, and the pressure is $101.325 \mathrm{kPa}$.

3) Wall conditions: both sides of the flow field are symmetrical boundaries; the solar panel and the upper and lower parts of the solar panel are wall surfaces; the roughness of the wall conditions is set as smooth. No slip wall condition is used.

\section{2 simulation setting of structure stress and modal analysis}

When the structural stress analysis of the solar support system is carried out, the flow field region needs to be restrained first. The following pre-processes are done on the supporting structure in ANSYS Workbench: defining material attributes, meshing, adding constraints and applying loads.

1) Defining material properties. The supporting part of the solar generator system is made of structural steel, the photovoltaic glass panel is made of toughened glass, and the solar panel frame is made of aluminum alloy. The material properties are shown in Table 1.

2) Meshing. The mesh of each part is done separately in the Workbench with reasonable sizes of surface mesh, volume mesh and line mesh.

3) Adding constraints. The structural steel bolt holes and bottom end faces at the bottom of the column of the solar support system are imposed with fixed constraints, and the whole solar panel supporting system is regarded as a rigid connection.

4) Applying loads. The solar panel supporting system is subjected to the loads of its own gravity and wind pressure. The gravity direction is vertical downward, and the pressure is the total wind pressure from the front and back of the solar panels. Moreover, the wind loads of the solar panel are different under different working conditions. The wind pressure data of solar panels under different working conditions obtained through fluid analysis is imported into the static structure analysis to obtain the stress and strain results of the solar panel supporting system.

Table 1. Material properties

\begin{tabular}{c|c|c|c|c}
\hline Materials & $\begin{array}{c}\text { Density/ } \\
\left(\mathrm{kg} / \mathrm{m}^{3}\right)\end{array}$ & $\begin{array}{c}\text { Elasticity } \\
\text { modulus/Gpa }\end{array}$ & $\begin{array}{c}\text { Poisson's } \\
\text { ratio }\end{array}$ & $\begin{array}{c}\text { Ultimate } \\
\text { strength/Mpa }\end{array}$ \\
\hline $\begin{array}{c}\text { Structural } \\
\text { steel }\end{array}$ & 7850 & 200 & 0.3 & 460 \\
\hline $\begin{array}{c}\text { Photovoltaic } \\
\text { glass }\end{array}$ & 2560 & 72.0 & 0.2 & 41.4 \\
\hline $\begin{array}{c}\text { Aluminum } \\
\text { alloy }\end{array}$ & 2770 & 71.0 & 0.33 & 310 \\
\hline
\end{tabular}

\section{Results and Analysis}

\subsection{Analysis of fluid simulation results}

According to the numerical simulation method and boundary conditions mentioned above, four different angles of winds with the same velocity $(41 \mathrm{~m} / \mathrm{s})$ are analyzed. The angles formed between the wind direction and the solar panel surface are $15^{\circ}, 63^{\circ}, 75^{\circ}$ and $165^{\circ}$ respectively. And the fluid numerical simulations of the solar panel supporting system under different working conditions are implemented and the comparison of wind load pressures on solar panels is showed in Fig.2, and comparison of average pressures showed in table 2 . 


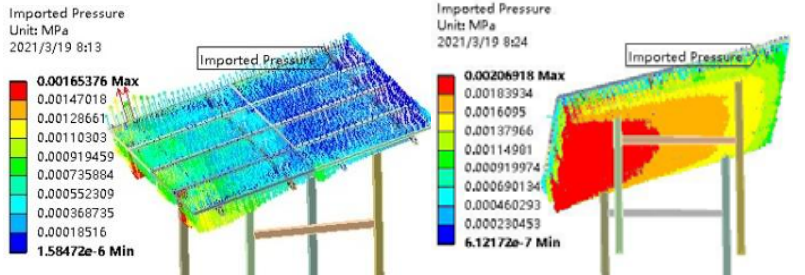

(a)

(b)

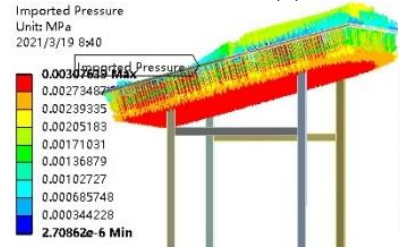

(c)

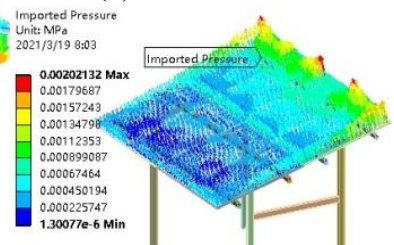

(d)

Fig.2. the pressure loads on solar panels of different directions winds; (a) $15^{\circ}$; (b) $63^{\circ}$; (c) $75^{\circ}$; (d) $165^{\circ}$

Table 2. Pressure comparison of different winds

\begin{tabular}{c|c|c|c|c}
\hline Wind direction & $15^{\circ}$ & $63^{\circ}$ & $75^{\circ}$ & $165^{\circ}$ \\
\hline Max pressure $\mathrm{Pa}$ & 1653.7 & 2069.2 & 3076.4 & 2021.3 \\
\hline Ave pressure $/ \mathrm{Pa}$ & 154.3 & 659.3 & 1695.2 & 91.4 \\
\hline
\end{tabular}

According to figure 2 and table 2, the difference between pressures produced by winds of different wind directions and same velocities is big. And the maximum pressure which is $0.00308 \mathrm{Mpa}$ is generated by the wind which is perpendicular to the solar panels, and the wind pressure distribution of it is relatively uniform on the entire solar panel. The average wind pressure $(0.00169$ Mpa) on solar panels is 3 to 20 times as big as that of other conditions. Therefore, the load of solar panels varies greatly with different wind directions. In the design, the main consideration should be given to the condition that the wind speed is perpendicular to the solar panels, which provides a useful theoretical reference for practical engineering.

\subsection{Stress-strain analysis of the supporting system}

Based on results of the fluid-structure interaction simulation, the wind pressures on the solar panel from the four different wind directions are different in values and distribution. The static stress-strain nephograms under different wind loads are shown in Fig. 3, and comparison of the static stress-strain values is shown in Table 3.

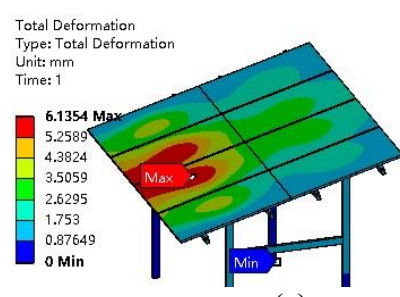

(a)

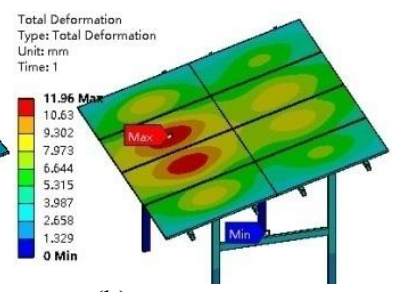

(b)

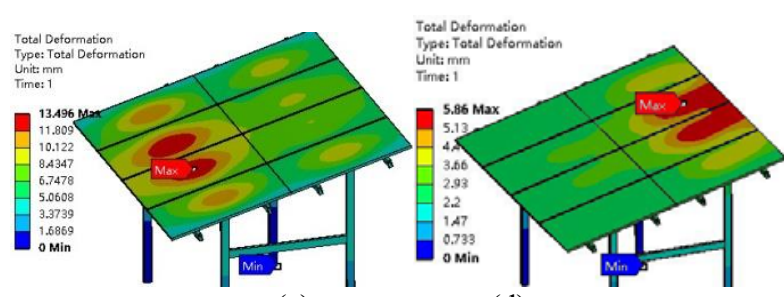

(c)

(d)

Fig. 4 static stress-strain nephogram of different wind loads: (a) $15^{\circ}$; (b) $63^{\circ}$; (c) $75^{\circ}$; (d) $165^{\circ}$

Table 3. Stress-strain values of different winds

\begin{tabular}{c|c|c|c|c}
\hline Wind direction & $15^{\circ}$ & $63^{\circ}$ & $75^{\circ}$ & $165^{\circ}$ \\
\hline Max Stress $/ \mathrm{Mpa}$ & 158.9 & 254.0 & 504.7 & 126.1 \\
\hline Max Strain /mm & 6.14 & 11.96 & 13.50 & 5.86 \\
\hline
\end{tabular}

According to the figure 3 and table 3 , it can be seen that the wind $\left(75^{\circ}\right)$ which is perpendicular to the solar panel has the greatest impact on solar panel system. And the maximum stress in the solar panel supporting system is $504.7 \mathrm{MPa}$, which is larger than the ultimate stress of the structural steel, which should be paid more attention to and optimized. The stresses generated in the other three working conditions $\left(15^{\circ}, 63^{\circ}, 165^{\circ}\right)$ all meet the allowable stress of the selected materials. Moreover, the maximum strain is $13.5 \mathrm{~mm}$, and the minimum strain is $5.86 \mathrm{~mm}$. Therefore, the wind direction has a great difference in the strain effect of the flat tye solar panel supporting system. The most dangerous working condition should be considered in the design engineering.

\subsection{Modal analysis of the supporting system}

Modal analysis can provide theoretical basis for vibration analysis, vibration fault diagnosis and prediction of the structural system. The solar panel supporting system may be affected by the seismic source under both free load and external load. In this study, the free modal analysis of the solar panel supporting system is carried out firstly, and then the external load modal analysis based on fluid-structure interaction is carried out respectively under different wind loads. In Modal analysis, low order natural vibration modes have a greater influence on the vibration mode of structure compared with high order natural vibration modes, and they are crucial to the dynamic characteristics of structure. Therefore this project focuses on the first six order modal frequencies and vibration models of the solar panel supporting system. The results of modal natural frequencies of the first six order modals of the supporting system under the wind flow to load are shown in figure 4 and 5.

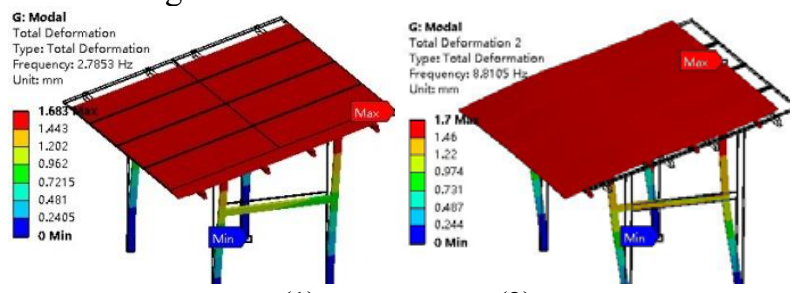

(1)

(2) 


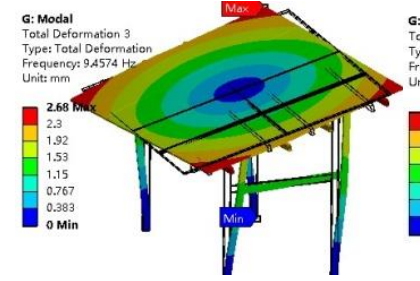

(3)

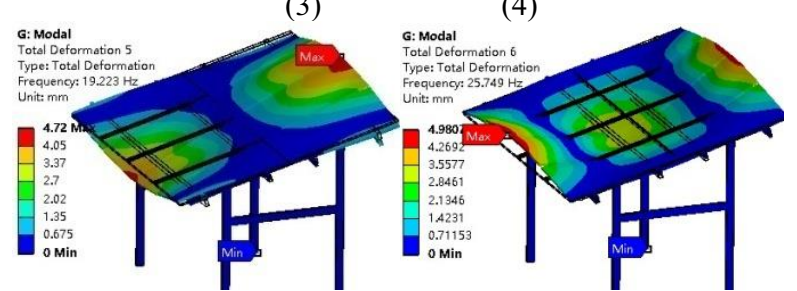

(5)

(6)

Fig.4. The first six order's natural frequency of solar support system; (1) order $1 ;(2)$ order 2 ; (3) order 3 ; (4) order 4 ; (5) order $5 ;(6)$ order 6

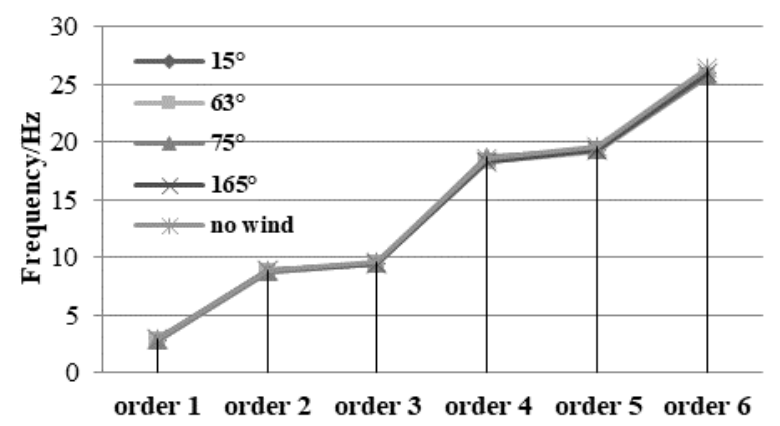

Fig.5. the first six frequencies of the solar support system

1) As it can be seen from Figure 4 and 5 , there is little difference in the variation trends between the free modal and the fluid-structure coupled pre-stress modals of the flat-plate solar panel supporting system. From the vibration shape figures of the first six modals of the system, it can be seen that the main vibrations of the system is appeared in pillar part of the supporting system. Both solar panels and pillar part of the supporting system experience certain amplitude in the third and fourth order modal analysis. In addition, the amplitude of solar panels is much more obvious in the fifth and sixth order modal analysis compared with the supporting part. Therefore, in the first three orders modal vibrations, the supporting part of the system should be focused mainly, and the vibration of solar panels should be paid attention to in the 4-6 order modal vibration.

2) According to the figure 4 and 5, the difference of the natural frequencies of the flat type solar energy system between the free modal and pre-load modal based on the fluid-structure interaction is small. However the inherent frequency of the free modal is slightly higher than that of pre-loaded modals. These results are consistent with the literatures [9].

3 ) In figure 5 , the natural frequencies of each order modal of the case of $63^{\circ}$ and $75^{\circ}$ are a little smaller than those of other cases, and the natural frequencies of each order modals of the solar panel supporting system are also similar between case $15^{\circ}$ and case $165^{\circ}$. Generally, the frequency of natural wind gust is low, so the wind directions of $63^{\circ}$ or $75^{\circ}$ should be considered primarily.

\section{Conclusions}

In this paper, the fluid simulation of the flat type solar supporting system under four typical wind directions with strong wind of level 13 is analyzed, and the wind pressure distribution of the solar panels is obtained. Then, the free mode and fluid-structure coupling modal analysis are carried out for the solar supporting system under no wind load and different wind loads, and the following conclusions are drawn through the analysis.

1) Wind directions and wind loads have great differences on the size and distribution of wind pressure of solar panels. The wind load perpendicular to the solar panel has the greatest influence on the stress-strain results of the solar system: the maximum stress is $504.7 \mathrm{MPa}$ which is already greater than the ultimate stress of structural steel. Therefore, the wind perpendicular to the solar panel should be focused in the design.

2) The variation trends of free modal and fluidstructure coupled modal analysis of the flat-plate solar supporting system have little difference. In the design, the supporting part of the system should be mainly considered in low order vibrations, while in high order vibrations, the vibration risk of the solar panel should be mainly considered.

3) The natural frequencies of the pre-loaded first six modals of the solar supporting system are all little lower than those in the free modal. In general, the frequency of natural wind gust is not high, so the results of the preloaded modal analysis should be considered in practical engineering applications to avoid resonance under external excitation.

\section{Acknowledgments}

The authors would like to thank the Science and Technology Ministry of China for supporting this science and technology partnership program project (Grant No. KY201702002), and thank the Cambodia Rural Development Agency for the cooperation of this project.

\section{References}

1. Sardeshpande V R, Chandak A G, Pillai I R. Procedure for thermal performance evaluation of steam generating point-focus solar concentrators[J].Solar Energy,2011,85(7):13901398.

2. Uematsu Y. Wind loads on free-standing canopy roofs: Part 2 overall wind forces [J].Journal of Wind Engineering and Industrial Aerodynamics, 2008, 96(6/7): 1029-1042.

3. Cheng Cheng, Xiaosheng Cheng, Ning Dai. Unidirectional Fluid-structure Coupling Analysis of Wind Turbine Blade $[\mathrm{J}]$. Chinese journal of Mechanical Design and Manufacturing Engineering, 2014,43( 11) : 28-31. 
4. Tomasini G, Cheli F. Admittance function to evaluate aerodynamic loads on vehicles: Experimental data and numerical model [J].Journal of Fluids and Structures, 2013,38:92-106. 2007,11(2/3):119-129.

5. Sohn M H, Chang J W. Flow visualization and aerodynamic load calculation of three types of clapfling motions in a weis-fogh mechanism [J]. Aerospace Science and Technology,

6. E Jianqiang, SU Xiuchao, WEN Ming Yang, et al. Journal of Hunan University (Natural Science Edition), 2015,42(02):8-16. (In Chinese)

7. B Kumar, B Raphael. CADREM: A Case -based System forConceptual Structural Design [J]. Engineering with Computers, 1997,13(3):153-164.

8. Li Huibin, Zhou Lilin, Sun Tiantian, et al. Journal of Vibration, Measurement \& Diagnosis, 2008,28 (3) :252- 255. (In Chinese)

9. Fang Zhihua, Zhang Weina, Yuan Yongqing. Journal of Chongqing University of Science and Technology, 2013,15 (2) :146-149. (In Chinese) 